\title{
GAMMA-RAY EMISSION FROM EXTRAGALACTIC RADIO SOURCES: EGRET OBSERVATIONS OF ACTIVE GALACTIC NUCLEI
}

\author{
PETER F. MICHELSON \\ Dept. of Physics, Stanford University \\ Stanford, Ca. 94305
}

\begin{abstract}
The Energetic Gamma-Ray Experiment Telescope (EGRET) on the Compton Gamma-Ray Observatory is an imaging high-energy telescope with sensitivity from approximately $20 \mathrm{MeV}$ to $30 \mathrm{GeV}$. EGRET has observed more than 129 sources during more than 4 years of operation. Among these sources, 51 have been identified with active galaxies. A common characteristic of the AGN sources is that they are all radio-loud, flat radio spectrum sources. Many of them are seen as superluminal radio sources as well. The gamma-ray emission characteristics of these sources are reviewed and some of the proposed emission models are discussed.
\end{abstract}

\section{Introduction.}

The Energetic Gamma Ray Experiment Telescope (EGRET) Instrument Team has reported the detection of 129 sources in the Second EGRET Catalog (Thompson et al. 1995). Figure 1 shows the positions of these sources in galactic coordinates. The size of the symbol for each source is proportional to the highest intensity seen for the source by EGRET. These sources include galactic pulsars, a nearby galaxy (the LMC), 51 active galaxies, several high-energy gamma- ray bursts, and 71 other sources not yet identified with known objects. A common characteristic of the AGN sources is that they are all radio-loud, flat radio spectrum sources (von Montigny et al. 1995). Many of them are seen as superluminal radio sources as well. They all appear to be members of the blazar class of AGN (Bl Lac objects, HPQ and OVV quasars), exhibiting one or more of the characteristics of this 
source class. One source, Mkn 421, has also been detected by the Whipple telescope in the $\mathrm{TeV}$ energy band (Punch et al. 1992). In addition more than two dozen bright, unidentified high-latitude sources have been detected.

Among the most interesting results obtained from analysis of the EGRET AGN observations are the following:

(i) the gamma ray energy flux in many of the sources is dominant over the emission in lower energy bands;

(ii) these sources are distributed over a wide range of redshifts (0.03 to 2.17);

(iii) the photon spectra in the EGRET energy range are generally wellrepresented by power laws in energy with a wide range of photon spectral indices $(-1.6$ to -2.6$)$;

(iv) many of the sources exhibit variability, in some cases the gamma-ray flux varies by a factor of 3 in less than a week;

(v) many active galaxies relatively close to earth, including Seyfert galaxies and some notable blazars and superluminal sources, have not been detected in high-energy gamma rays.

\section{Gamma-ray emission models.}

There are several arguments suggesting that the gamma-ray emission from the EGRET sources is strongly beamed. In particular, the EGRET observations of high fluxes above $1 \mathrm{GeV}$, short timescale variability, and consideration of absorption via photon-photon pair production appear to rule out emission models in which the gamma radiation is generated in the same volume as the observed $\mathrm{X}$-ray radiation, unless the radiation is beamed towards us (McBreen 1979). This is consistent with the interpretation of blazars as objects in which the non-thermal radiation arises predominantly from a "jet" of relativistic particles directed close to the observer's line of sight (Blandford and Rees 1978). However, the gamma-ray emission in most of the EGRET sources cannot come from radii as large as VLBI sources because of variability constraints. Thus the gamma emission is an important link between radio structure and the putative black hole central engine.

If the gamma-ray emission is from a source moving relativistically with velocity $\beta c$ and having bulk Lorentz factor $\Gamma=\left(1-\beta^{2}\right)^{-1 / 2}$, then a local observer, whose line of sight is at an angle $\Theta$ to the direction of the bulk motion, will see emission with apparent luminosity $L_{a p p}$ and duration $\Delta t_{a p p}$ given by

$$
L_{\text {app }}=L \delta^{p}, \Delta t_{\text {app }}=\Delta t \delta^{-1},
$$

where $\delta=[\Gamma(1-\beta \cos \Theta)]^{-1}, L$ is the comoving luminosity, $\Delta t$ is the comoving time duration of the emission, and $p$ is typically between 3 and 4 


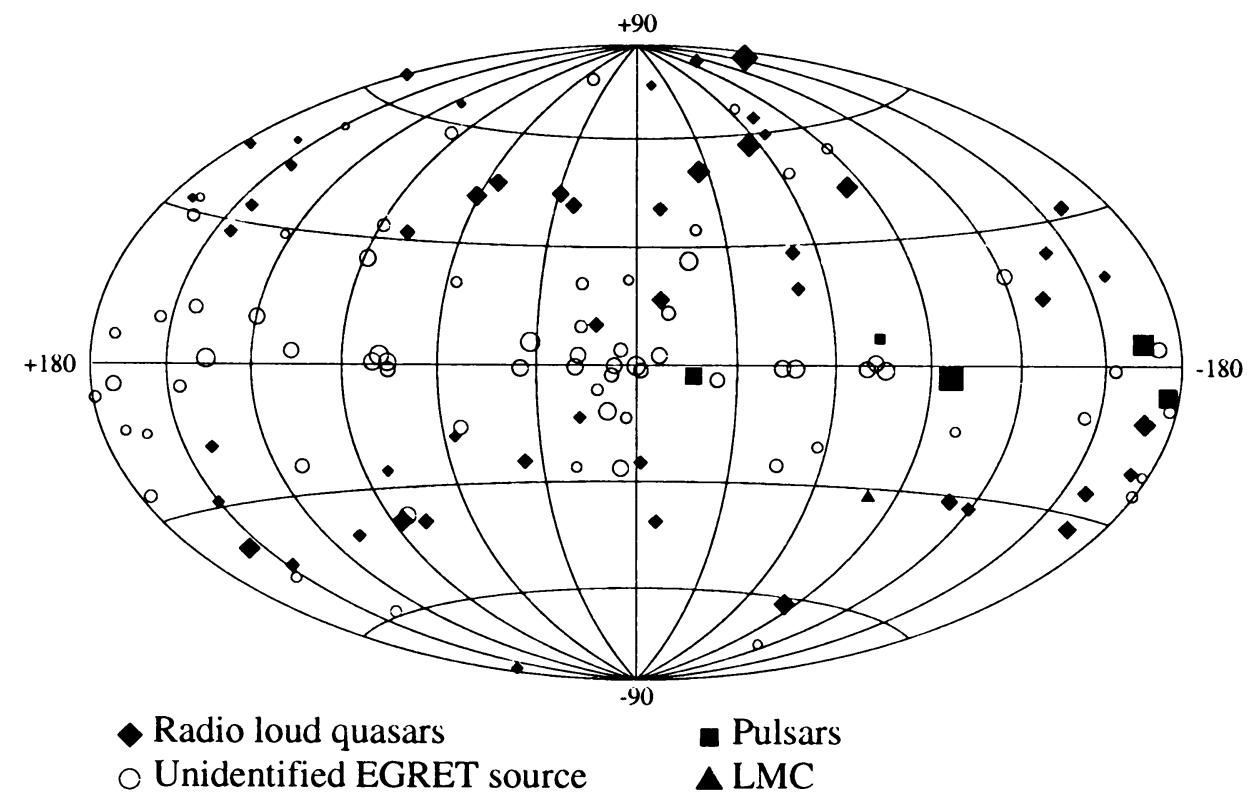

Figure 1. The second EGRET catalog of high-energy gamma-ray sources.

(Lind and Blandford 1985). Reasonable values of $\delta$ result in gamma-gamma opacities near unity. Interestingly, relativistic beaming may also play an important role in gamma-ray bursts. In particular EGRET and COMPTEL observations of high-energy emission from some relatively short duration gamma-ray bursts appears to require that the sources are strongly beamed with \& approaching 1000 if the bursts are cosmological.

Details of models for the AGN gamma-ray emission are as varied as those for the sub-MeV emission, with most involving inverse Compton scattering of low energy photons up to gamma-ray energies by beamed relativistic electrons. The low energy photons come from either a disk (Melia and Königl 1989; Dermer et al. 1992) or are self-generated by the electrons as synchrotron radiation (Jones, O'Dell, and Stein 1974; Marscher 1980; Königl 1981; Marscher and Bloom 1992; Maraschi et al. 1992). It has also been proposed (Blandford and Levinson 1995; Sikora, Begelman, and Rees 1994) that radiation from a disk which has been reprocessed in a halo (eg., broad-line region clouds, disk wind, dust) may be more effectively Compton scattered than radiation coming directly from the disk. This is because in the comoving frame of the gamma-ray producing plasma, the unscattered disk radiation is strongly redshifted while diffuse reprocessed radiation is strongly blueshifted as the jet passes near or through the reprocessing region.

In contrast to the leptonic jet models, the proton-initiated cascade (PIC) 
model of Mannheim and Biermann $(1989,1992)$ is based on shock acceleration of protons to energies so high $\left(10^{7}-10^{8} \mathrm{TeV}\right)$ that the threshold for photomeson production is exceeded and that the proton cooling timescale becomes equal to the electron cooling timescale. The PIC model has been applied to the spectra of 3C 273, 3C 279, and Mkn 421 (Mannheim 1993). Generally, the model can account for the wide range of spectral indices observed in the EGRET energy range and predicts that below a few $\mathrm{MeV}$, the photon spectrum in the X-ray range should flatten.

In the emission models involving inverse Compton scattering by relativistic electrons, the relative importance of self-Compton scattering and inverse Compton reflection depends on the ratio of the internally generated synchrotron photon density to the ambient photon density, as measured in the rest frame of the jet plasma. Thus, depending on the conditions, either self-Compton scattering or inverse Compton scattering of photons emitted by the accretion disk (and possibly scattered back toward the jet by an ambient medium) might be the dominant gamma-ray emission process. Since all of these photon fields are expected to be present within $\sim 1 \mathrm{pc}$ of the central engine, the relative importance of each depends on the value of various parameters such as the location of the Compton scattering region and the ratio of apparent synchrotron luminosity to luminosity of the accretion disk.

\section{References}

Blandford, R.D. \& Levinson, A. 1995, ApJ, 441, 79

Blandford, R. \& Rees, M.J., 1978, in Pittsburgh Conference on Bl Lac Objects, ed. A.N. Wolfe (University of Pittsburgh Press), 328

Dermer, C.D., Schlickeiser, R., \& Mastichiadis, A. 1992, A\&A, 256, L27

Dermer, C.D., \& Schlickeiser, R. 1992, Science, 257, 1642

Jones, T.W., O'Dell, S.L., \& Stein, W.A. 1974, ApJ, 188, 353

Königl, A., 1981, ApJ, 243, 700

Lind, K.R., and Blandford. R.D., 1985, ApJ, 295, 358

Mannheim, K., \& Biermann, P.L., 1992, $A \& A$, 53, L21

Mannheim, K., \& Biermann, P.L., 1989, $A \& A, 221,211$

Mannheim, K., 1993, Phys. Rev. D, 48, 2408

Maraschi, L., Ghisellini, G., \& Celotti, A., 1992, ApJ, 397, L5

Marscher, A.P., 1980, ApJ, 235, 386

Marscher, A.P., \& Bloom, S.D., 1992, in Proc. of the CGRO Science Workshop, NASA Conf. Pub. 3137, 346

McBreen, B., 1979, $A \& A, 71, \mathrm{~L} 17$

Melia, F., \& Königl, A., 1989, ApJ, 340, 162

von Montigny, C., et al., 1995, ApJ, 440, 525

Punch, M., et al., 1992, Nature, 358, 477

Sikora, M., Begelman, M.C., \& Rees, M.J. 1993, in Proceedings of the Compton Symposium, in press

Thompson, D.J., et al., 1995, The Second EGRET Catalog of High-Energy Gamma-Ray Sources, accepted for publication in ApJ Suppl. 